Research Paper

\title{
Immunomodulatory Effects of Newcastle Disease Virus AF2240 Strain on Human Peripheral Blood Mononu- clear Cells
}

Han Yuen Lam ${ }^{1}$, Khatijah Yusoff ${ }^{1}$, Swee Keong Yeap², Tamilselvan Subramani ${ }^{1}$, Suraini Abd-Aziz ${ }^{1}$, Abdul Rahman Omar ${ }^{2}$, Noorjahan Banu Alitheen ${ }^{1 凶}$

1. Faculty of Biotechnology and Biomolecular Sciences, Universiti Putra Malaysia, 43400, Serdang, Selangor, Malaysia.

2. Institute of Bioscience, Universiti Putra Malaysia, 43400, Serdang, Selangor, Malaysia.

$\triangle$ Corresponding author: E-mail: noorjahan@upm.edu.my

( ) Ivyspring International Publisher. This is an open-access article distributed under the terms of the Creative Commons License (http://creativecommons.org/ licenses/by-nc-nd/3.0/). Reproduction is permitted for personal, noncommercial use, provided that the article is in whole, unmodified, and properly cited.

Received: 2013.1I.19; Accepted: 2014.07.21; Published: 2014.09.19

\begin{abstract}
Immunotherapy has raised the attention of many scientists because it hold promise to be an attractive therapeutic strategy to treat a number of disorders. In this study, the immunomodulatory effects of low titers of Newcastle disease virus (NDV) AF2240 on human peripheral blood mononuclear cells (PBMC) were analyzed. We evaluated cytokine secretion and PBMC activation by cell proliferation assay, immunophenotyping and enzyme linked immunosorbent assay. The proliferation of the human PBMC was measured to be $28.5 \%$ and $36.5 \%$ upon treatment with 8 hemaglutinin unit (HAU) and $2 \mathrm{HAU}$ of NDV respectively. Interestingly, the percentage of cells with activating markers CDI6 and CD56 were increased significantly. Furthermore, the intracellular perforin and granzyme levels were also increased upon virus infection. Human PBMC treated with NDV titer $8 \mathrm{HAU}$ was found to stimulate the highest level of cytokine production including interferon- $\gamma$, interleukin-2 and interleukin- 12 . The release of these proteins contributes to the antitumor effect of PBMC against MCF-7 breast cancer cells. Based on the 3-(4, 5-dimethylthiazol-2-yl)-2, 5-diphenyl tetrazolium bromide assay, activated human PBMC showed high cytolytic efficiency towards human breast tumor cells. In summary, NDV was able to stimulate PBMC proliferation, cytokine secretion and cytolytic activity.
\end{abstract}

Key words: Newcastle disease virus; AF2240; immunomodulation; PBMC; breast cancer; immunophenotyping

\section{Introduction}

Immunotherapy refers to the treatment of a disease by strengthening the body's immune system. To enhance the immune system, immunostimulatory agents such as chemicals, proteins, and viruses are needed [1]. Newcastle disease virus (NDV) is classified into the family of Paramyxoviridae and genus Avulavirus [2]. According to Alexander and Jones [3], NDV can be further divided into three pathotypes based on the virulence and severity of the disease, which are velogenic (highly contagious), mesogenic (moderate) and lentogenic (mild infection). In addition to its adverse effects on avian species, NDV has been discovered to have oncolytic effects on human tumor cells [4]. Sturlan et al. [5] showed that activated human peripheral blood mononuclear cells (PBMC) exerted a cytotoxic effect on melanoma, colon cancer and breast carcinoma, upon influenza A virus infection. Their findings suggested the potential of using virus to enhance the anticancer activity of the human immune system. In fact, NDV had been found to stimulate the production of cytokines, such as interferon (IFN)- $\alpha$, IFN- $\beta$, tumor necrosis factor (TNF)- $\alpha$ and interleukin (IL)-1, which in turn leads to the activation of natural killer (NK) cells, macrophages, and sensitized T cells $[6,7]$.

Report from Biron et al. [8] described that vi- 
rus-induced IFN- $\alpha$ and IFN- $\beta$ are potent inducers of NK cell-mediated cytotoxicity and that NK cells are important contributors to innate defense against viral infections. In this study immunophenotyping of the activated immune cells were performed to determine the activation of subpopulations in human PBMC, especially CD56 and CD16 immune cells, such as NK cells which play important roles in cell-killing mechanisms. In addition, cytokines secretion by activated immune cells, especially T-helper (TH) 1 , is another measurement of immunostimulatory effect. TH1 cytokines include IFN- $\gamma$, IL-2 and IL-12. These cytokines can augment proliferation and cytotoxic effects of human PBMC [9-11]. Hence, quantification of extracellular cytokines which were secreted into the cell culture supernatant suggested the activation state of the cytolytic effector cells including NK cell and cytolytic T cell. Activated lymphocytes and NK cells are designed to protect the host from invasion of pathogens and tumor cells. They induce apoptosis on the tumor target using granule exocytosis, Fas / Apo-1 and TNF- $\alpha$ dependent process. The granule exocytosis pathway involves perforins that form pores on the cell membrane of the target cell allowing the granzymes to enter the cells, inducing apoptosis [12]. To determine the production of granzymes and perforin, an intracellular staining method was used to access the activation of lymphocytes and NK cells. Thus, we hypothesised that the levels of intracellular perforin and granzymes may reflect the activity of activated lymphocytes and NK cells. To our best knowledge, the immunomodulatory effects of NDV strain AF2240 on human lymphocytes have yet to be studied. Hence, in this study, the immunostimulatory effects of human PBMC by NDV AF2240 velogenic strain were analyzed.

\section{Materials and Methods}

\section{I. Newcastle disease virus}

The local NDV fowl/Malaysia/AF2240 (AF048763) strain was used throughout this study. It has been found to have an intravenous pathogenicity index (IVPI) of 2.56, intracerebral cytopathogenicity index (ICPI) of 1.9, mean death time (MDT) of 53 hours and 105 EID506. This velogenic virus strain can cause $100 \%$ mortality in susceptible flocks [10].

\subsection{Virus propagation and purification}

The NDV AF2240 strain was propagated in allantoic fluid of 10 days-old embryonated chicken eggs at $37^{\circ} \mathrm{C}$ [11]. After 3 days, the allantoic fluids were harvested and spun down by high speed centrifugation. The virus stock was further purified by sucrose gradient ultracentrifugation. The virus stock was resuspended with phosphate buffer saline (PBS) and stored at $-20^{\circ} \mathrm{C}$. Hemaglutination test was carried out to determine the purified virus titer in hemaglutinin unit (HAU). Purified virus contained 128HAU titer.

\subsection{Isolation of PBMC}

Blood $(20-25 \mathrm{~mL})$ was taken at the Universiti $\mathrm{Pu}-$ tra Malaysia Student Health Center from 15 healthy donors (7 female and 8 male) using a $25 \mathrm{ml}$ syringe and kept in vacutainer with sodium heparin (BD, USA). The donor's health condition was evaluated by the medical official at the same and each subject provided written informed consent according to the requirements of the Ethics Committee of Biomedical Research of Universiti Putra Malaysia and the principles expressed in the Helsinki Declaration. The blood samples were mixed with the same volume of PBS. The diluted blood sample was layered on Ficoll-Paque Plus (Amersham Biosciences, USA) by using a Pasteur pipette. The mixture was centrifuged under $400 \mathrm{x} g$ for 40 minutes at $18-20^{\circ} \mathrm{C}$. After centrifugation, the lymphocyte layer was transferred to a new tube and washed twice with PBS. The cell pellet was resuspended with Roswell Park Memorial Institute (RPMI)-1640 media (Sigma, USA) with 10\% fetal bovine serum (FBS) (PAA, Austria). The trypan blue dye exclusion assay was performed to determine the cell number and the cell viability of PBMC.

\subsection{5-bromo-2'-deoxyuridine (BrdU) assay}

Cell proliferation assay was performed by using the BrdU Cell Proliferation Assay kit (Calbiochem, USA) according to the manufacturer's instruction. The PBMC were cultured in the 96-well tissue culture microtiter plates (Nunc, Denmark) and incubated for 24, 48 and 72 hours, respectively, at $37^{\circ} \mathrm{C}$ and $5 \% \mathrm{CO}_{2}$. After the corresponding period, BrdU label was added into all wells and incubated for an additional 24 hours. Then, fixative/denaturing solution was loaded to fix the cells on the plate for 30 minutes followed by addition of anti-BrdU antibody into the wells. The wells were washed thrice with PBS to remove the non-specific binding and then the conjugate was added into the wells. After 30 minutes, the 3,3',5,5'-Tetramethylbenzidine (TMB) substrate solution was loaded in dark condition. After adding the $0.16 \mathrm{M}$ sulfuric acid stop solution, the plate was read by $\mu$-Quant ELISA Reader (Bio-Tek Instruments, USA) at the wavelength of 450-540nm [12]. Percentage of cell proliferation was calculated using the following formula:

$$
\% \text { of cell proliferation }=\frac{\text { OD sample }}{\text { OD control }} \times 100
$$

\subsection{Immunophenotyping}

Flow cytometric immunophenotypingwas uti- 
lized to determine the expression level of surface marker such as CD56 and CD16 on the activated lymphocyte and to determine the level of perforin and granzymes that assembled in the cells. All the human targeting monoclonal antibodies were purchased from Dako, Denmark. Human PBMC were exposed to virus titer 8 and $2 \mathrm{HAU}$, individually for 72 hours. The cells were harvested and washed twice with PBS by centrifugation $(200 x g)$. The cells were stained with either anti-CD16 (FITC) or anti human CD56 antibody conjugated with phycoerythrin (PE) and analyzed by BD FACS Calibur multicolor flow cytometer (Becton, Dickinson and Co., USA).To measure the level of perforin and granzymes in the cells, intracellular double stain immunophenotyping was conducted. The PBMC were fixed and permeabilized with the aid of Cytofix/CytopermTMFixation/Permeabilization Kit (BD Biosciences, USA). After treating the PBMC with the NDV for 72 hours, the cells were harvested. Then, the cells were stained with fluorochrome-conjugated monoclonal anti-human CD56 antibody targeted to the lymphocytes and NK cells. The cells were washed twice to remove the non-specific binding and then mixed with Fixation/Permeabilization solution for 20 minutes. The fixed cells were washed with Perm/WashTM buffer and then stained again with fluorochrome-conjugated monoclonal antibody specific for the desired cytokines or proteins. The cells were analyzed by BD FACS Calibur multicolor flow cytometer (Becton, Dickinson and Co., USA).

\subsection{Enzyme-linked immunosorbent assay}

The targeted cytokine (IFN- $\gamma$ ) was analyzed using the Instant ELISA kit (Bender MedSystems, Austria) based on suppliers protocol. Upon NDV treatment with PBMC,the supernatant was collected and loaded in antibody pre-coated microplate for 3 hours. The plate was washed with wash buffer and the TMB substrate solution was added in the wells. After adding the stop solution, the absorbance was read on a $\mu$-Quant ELISA Reader (Bio-Tek Instruments, USA) using $450 \mathrm{~nm}$ wavelength and $620 \mathrm{~nm}$ reference wavelength [12].The level of granzyme B was analyzed using Granzyme B ELISA kit (Bender MedSystems, Austria) in the supernatant of PBMC co-cultured with MCF-7 at the ratio of effector cell (PBMC) to target cancer cell (MCF-7) of 5:1 for 24 hours.

\subsection{3-(4, 5-dimethylthiazol-2-yl)-2, 5-diphenyl tetrazolium bromide (MTT) microcytotoxicity assay}

The MCF-7 cells (target) were seeded in 96-well tissue culture plates (Nunc, Denmark) and incubated for 24 hours at $37^{\circ} \mathrm{C}$ and $5 \% \mathrm{CO}_{2}$. NDV-treated (titer 8
HAU, titer 2 HAU) and non-treated negative control PBMC were cultured for 72 hours in 6-well tissue culture plates (Nunc, Denmark). After the incubation period, the PBMC were washed with PBS to remove the virus. The treated PBMC were co-cultured with MCF-7 cells in the ratio of effector cell (PBMC) to target cancer cell (MCF-7) of 5:1, 2:1 and 1:1 for 24 hours. The media from all wells was removed and washed 3 times with PBS and replaced with fresh media to remove the effector PBMC and detached MCF-7 cell. The removed media was pelleted and the supernatant was subjected to hemaglutination test to evaluate the present of virus residue in the culture. No HAU was detected from all the harvested media. On the other hand, MTT solution (Sigma, USA) was added into the wells and incubated again for 4 hours to evaluate the viability of the attached and healthy MCF-7 cell. Dimethyl sulfoxide (DMSO) (Fisher scientific, UK) was replaced into each well to dissolve the formazan crystal formed and the plate was read by $\mu$-Quant ELISA Reader (Bio-Tek Instruments, USA) at wavelength $570 \mathrm{~nm}$.

\subsection{Annexin-V-FITC/PI apoptosis assay}

NDV-treated (titer $8 \mathrm{HAU}$ or titer $2 \mathrm{HAU}$ ) and non-treated PBMC were cultured for 72 hours in 6-well tissue culture plates (Nunc, Denmark). Later, the PBMC was co-cultured with MCF-7 in the ratio of effector cell (PBMC) to target cancer cell (MCF-7) of 5:1 for 24 hours.After incubation, all the suspended cellswere discard and the adherent MCF-7 cells werestained withAnnexinV-FITC/PI apoptosis kit (BD, USA) and analyzed by BD FACS Calibur multicolor flow cytometer (Becton, Dickinson and Co., USA).

\section{Statistical analysis}

The results were analyzed using the SPSS Statistics 17 software. The results were expressed as mean \pm standard error (SE). The significance of differences between means was determined using One Way ANOVA test with Duncan test. $\mathrm{p} \leq 0.05$ was taken as statistically significant.

\section{Results}

\section{I. Cell proliferation assessment of activated PBMC upon NDV treatment}

Cell proliferation was investigated to determine the immunostimulatory effects of NDV using BrdU cell proliferation assay by measuring the incorporation of thymidine analog Bromodeoxyuridine. BrdU is incorporated into the newly synthesized DNA of replicating cells during the synthesis phase of the cell cycle, indicating that the cells are actively replicating 
their DNAs. Exposure to NDV by human PBMC increased the cell proliferation by $28 \pm 7.329 \%$ and $36 \pm 13.033 \%$ after being treated with virus titer 8 HAU and 2 HAU respectively for 72 hours compared to untreated control cells (Figure 1). These results clearly reflected the increase of proliferation potency upon virus treatment.

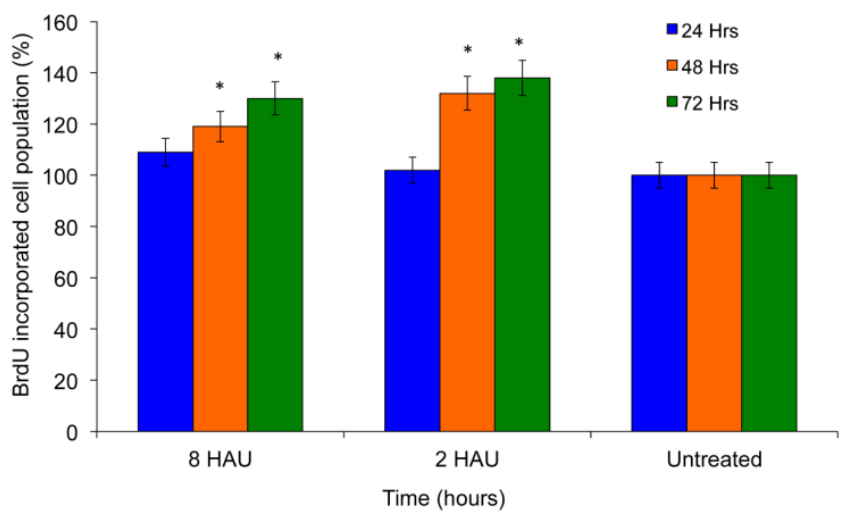

Figure I. BrdU cell proliferation study of human peripheral blood mononuclear cells (PBMC) after exposed to NDV for 72 hours. The values were the means \pm SE of three independent experiment. The differences between the control group and treated group were determined by one-way ANOVA. $(* \mathrm{p}<0.05)$.

\subsection{Immunophenotyping analysis of activated PBMC}

In this study, the activated PBMC were stained with anti-human CD56 and anti-human CD16 antibodies and analyzed by flow cytometry and the results are shown in Table 1 . The population of cytolytic NK cells that expressed signals to CD56 and CD16, were increased significantly to $9.163 \pm 0.34 \%$ and $5.79 \pm 0.362 \%$ upon treatment with virus titer $8 \mathrm{HAU}$ and 2 HAU respectively compared to control $(4.31 \pm 0.144 \%)$. Similarly, the population of cytokine secreting NK cells was also increased, after exposure to NDV.

Table I. FACS immunophenotyping analysis of activated human PBMC by NDV for 72 hours by staining with anti-human CD56 and CDI 6 monoclonal antibody. The values were the means \pm SE of three independent experiments. The data were analyzed for significance by SPSS $(p<0.05)$.

\begin{tabular}{|c|c|c|c|c|}
\hline \multirow[t]{3}{*}{ Virus titer } & \multicolumn{2}{|c|}{ CD56- } & \multicolumn{2}{|c|}{$\mathrm{CD}^{2} 6^{+}$} \\
\hline & CD16- $^{-}$ & $\mathrm{CD}^{2} 6^{+}$ & CD16- & $\mathrm{CD} 6^{+}$ \\
\hline & Others & $\begin{array}{l}\text { Macrophages } \\
\text { Neutrophils }\end{array}$ & $\begin{array}{l}\text { Cytokine se- } \\
\text { creting NK cells }\end{array}$ & $\begin{array}{l}\text { Cytolytic NK } \\
\text { cells }\end{array}$ \\
\hline $8 \mathrm{HAU}$ & $79.920 \pm 0.817^{*}$ & $1.690 \pm 0.199$ * & $9.220 \pm 0.450$ * & $9.163 \pm 0.340$ * \\
\hline $2 \mathrm{HAU}$ & $84.830 \pm 0.766$ * & $1.007 \pm 0.253$ & $8.373 \pm 0.215$ * & $5.790 \pm 0.362$ * \\
\hline $\begin{array}{l}\text { Untreated } \\
\text { PBMC }\end{array}$ & $92.050 \pm 0.242$ & $0.363 \pm 0.055$ & $3.277 \pm 0.093$ & $4.310 \pm 0.144$ \\
\hline
\end{tabular}

\subsection{Cytokines secretion analysis upon NDV induction}

Figure 2 shows the level of cytokines secreted after NDV treatment. It has been demonstrated that NDV is a potent inducer of IFN- $\gamma$ and increasing level of IFN- $\gamma$ might reflect the activation status of human PBMC [13]. Many reports showed the ability of IFN- $\gamma$ activating and enhancing the immune cells including NK cells, T lymphocytes and macrophages [8,12,14]. After 48 hours of NDV treatment, the highest level of IFN- $\gamma$ was observed $(44.221 \pm 0.903 \mathrm{pg} / \mathrm{ml})$ at titer 8 HAU (Figure 2); and the cells treated with NDV titer 2 $\mathrm{HAU}$, the IFN- $\gamma$ level was $27.566 \pm 4.159 \mathrm{pg} / \mathrm{ml}$ compared to the mock infected control $(8.752 \pm 1.699 \mathrm{pg} / \mathrm{ml})$.

The level of IL-2 was also determined as it has the ability to augment the immune functions of NK cells such as T lymphocytes and macrophages. Figure 3 shows the time dependent increase of IL-2 levels by human PBMC after NDV induction. The highest IL-2 level was obtained after 72 hours for both treatment groups. The level of IL-12 increased significantly ( $p<$ 0.05 ) after being cultured with NDV titer $8 \mathrm{HAU}$ and 2 HAU for all 24, 48 and 72 hours except treatment time 72 hours for treatment group with titer 2 HAU (Figure 4). We speculate that IL-12 plays a role in enhancing cytotoxicity of human immune system against cancer cell. Taken together, the release of augmented levels of cytokines indicated the activation status of human PBMC. These results complemented with the findings by Carlens et al. [15] and Ortaldo et al. [16] who reported that cytokines, such as IFN- $\gamma$, IL-2 and IL-12 might enhance NK cell proliferation and activities.

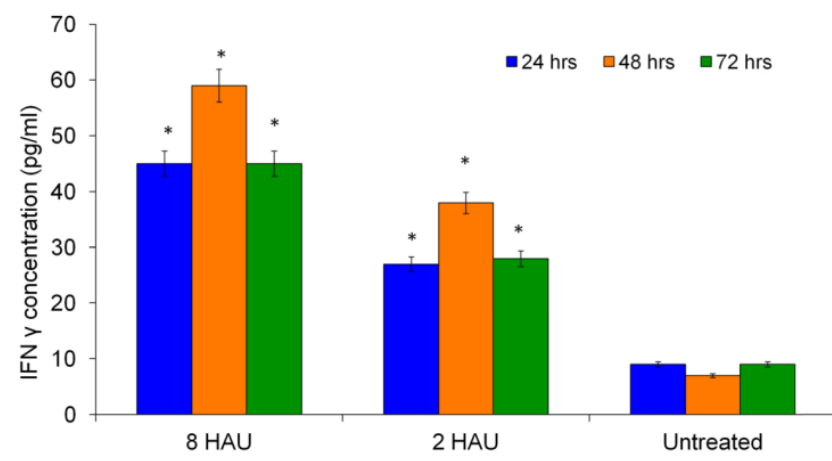

Figure 2. ELISA results for cytokine (IFN- $\gamma$ ) detection after human PBMC treated with virus $8 \mathrm{HAU}, 2 \mathrm{HAU}$ and control. The values were the means \pm SE of three independent experiment. The differences between the control group and treated group were determined by one-way ANOVA. $(* p<0.05)$. 


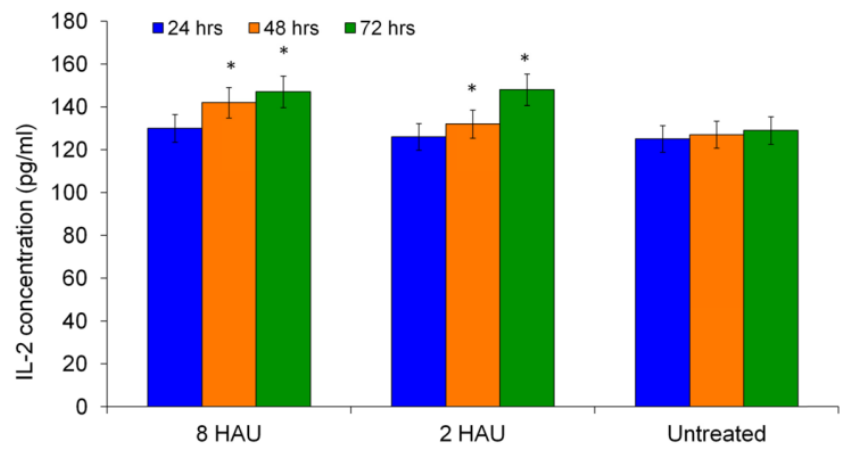

Figure 3. ELISA result showed the level of interleukin-2 (IL-2) released from the human PBMC after treated by NDV at different titers. The values were the means \pm $S E$ of three independent experiment. The differences between the control group and treated group were determined by one-way ANOVA. $(* p<0.05)$.

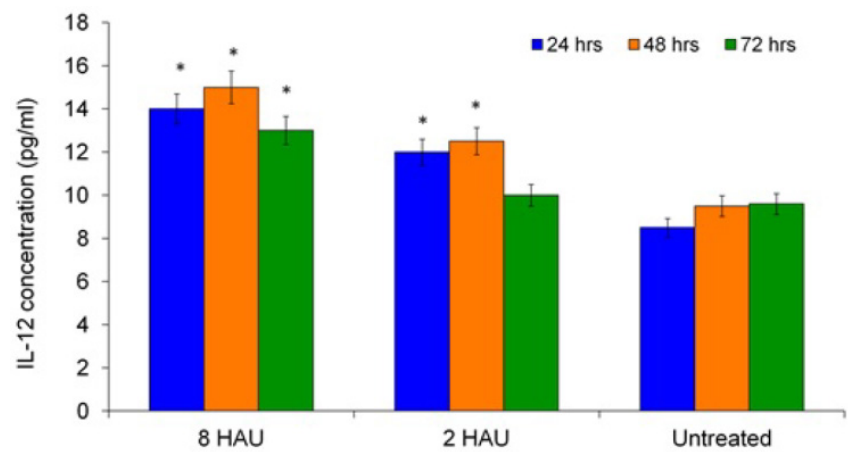

Figure 4. ELISA result showed the level of interleukin- 12 released from the human PBMC after treated by NDV at different titers. The values were the means \pm SE of three independent experiment. The differences between the control group and treated group were determined by one-way ANOVA. $(* \mathrm{p}<0.05)$.

\subsection{Expression of granules in NDV activated PBMC}

The intracellular protein, perforin, is involved in granule-mediated cytolytic mechanism. The level of intracellular perforin synthesised by activated PBMC was determined by immunophenotyping. The percentage of cells that contained perforin increased significantly after exposure to the virus for 72 hours (Table 2). The percentages of CD56 positive NK cells which contained perforin was significantly increased ( $\mathrm{p}<0.05$ ) from $4.28 \pm 0.143 \%$ (control) to $10.57 \pm 0.392 \%$ (treated by $8 \mathrm{HAU}$ virus) and $9.43 \pm 0.027 \%$ (treated by 2 HAU virus). On the other hand, the T cell population at $8 \mathrm{HAU}$ virus was able to induce significant changes of perforin synthesis compared to control. In addition, we analyzed the production of another intracellular granule protein, granzyme B that functions to induce apoptosis and cell lysis using flow cytometry immunophenotyping. The results showed that the population of cells containing granzyme B increased significantly upon virus treatment (Table 3 ). The populations gave fluorescence signals for both granzyme B and CD56 indicating the presence of NK cells. The percentage of NK cells was significantly increased $(p<0.05)$ to $10.53 \pm 0.391 \%$ and $9.11 \pm 0.026 \%$, after treatment with NDV at $8 \mathrm{HAU}$ and $2 \mathrm{HAU}$, respectively. Furthermore, the population of none-activated NK cell (CD56+Perforin-/Granzyme B-) was also found to be increased after being treated with 8 HAU and 2 HAU of NDV (Table 2 and Table 3 ).

Table 2. FACS immunophenotyping analysis of activated human PBMC by NDV for 72 hours by staining with Anti-human CD56 and perforin antibody. The values were the means \pm SE of three independent experiment. The data were analyzed for significance by SPSS $(p<0.05)$.

\begin{tabular}{lllll}
\hline Virus titer & \multicolumn{2}{c}{ CD56 $^{-}$} & \multicolumn{2}{c}{ CD56 $^{+}$} \\
\cline { 2 - 5 } & Perforin- & Perforin $^{+}$ & Perforin- & \multicolumn{1}{c}{ Perforin } \\
\cline { 2 - 5 } & Others & $\begin{array}{l}\text { T lympho- } \\
\text { cytes }\end{array}$ & $\begin{array}{l}\text { Non-activated NK cells } \\
\text { NK cells }\end{array}$ \\
\hline 8 HAU & $70.99 \pm 0.726^{*}$ & $5.80 \pm 0.683^{*}$ & $12.64 \pm 0.612^{*}$ & $10.57 \pm 0.392^{*}$ \\
2 HAU & $71.63 \pm 0.647^{*}$ & $2.23 \pm 0.560$ & $16.71 \pm 0.429 *$ & $9.43 \pm 0.027^{*}$ \\
Untreated & $85.59 \pm 0.225$ & $2.56 \pm 0.388$ & $7.57 \pm 0.215$ & $4.28 \pm 0.143$ \\
PBMC & & & & \\
\hline
\end{tabular}

Table 3. FACS immunophenotyping analysis of activated human PBMC by NDV for 72 hours by staining with Anti-human CD56 and granzyme $B$ antibody. The values were the means $\pm S E$ of three independent experiment. The data were analyzed for significance by the statistic software SPSS $(p<0.05)$.

\begin{tabular}{|c|c|c|c|c|}
\hline \multirow[t]{3}{*}{ Virus titer } & \multicolumn{2}{|c|}{ CD56- } & \multicolumn{2}{|c|}{$\mathrm{CD}^{2} 6^{+}$} \\
\hline & Granzyme B- & Granzyme B+ & Granzyme B- & Granzyme B+ \\
\hline & Others & $\begin{array}{l}\text { Cytolytic T } \\
\text { lymphocytes }\end{array}$ & $\begin{array}{l}\text { Non-activated } \\
\text { NK cells }\end{array}$ & NK cells \\
\hline $8 \mathrm{HAU}$ & $71.90 \pm 0.735$ * & $3.72 \pm 0.438^{*}$ & $13.85 \pm 0.670$ * & $10.53 \pm 0.391$ * \\
\hline 2 HAU & $72.88 \pm 0.658$ * & $3.52 \pm 0.884^{*}$ & $14.49 \pm 0.372$ * & $9.11 \pm 0.026$ * \\
\hline Untreated PBMC & $84.00 \pm 0.221$ & $2.69 \pm 0.408$ & $9.72 \pm 0.276$ & $3.59 \pm 0.120$ \\
\hline
\end{tabular}

\subsection{Cytolytic effects of activated PBMC on human breast cancer MCF-7 cells}

MTT assay was performed to determine the cytolysis effects of activated PBMC towards MCF-7 cell lines. After 72 hours of incubation of the human PBMC with NDV, the NDV was removed by washing with PBS and the activated PBMC cells were co-cultured with MCF-7 cells. We observed that the higher PBMC effector to MCF-7 target (E/T) ratio gave higher reduction of target cell viability (Figure 5); the MCF-7 viability dropped to $69.66 \%$ and $70.18 \%$ after being treated with activated PBMC by NDV at 8 and 2 HAU respectively at 5 to $1 \mathrm{E} / \mathrm{T}$ ratio. Furthermore, we carried out Annexin-V-FITC/PI flow cytometry apoptosis assay to determine the mode of cell death induced by NDV treated PBMC against MCF-7.The results showed that the activated PBMC induced around $30 \%$ of MCF-7 to undergo late apoptosis similar to the results of the MTT assay. At the same time, 8 HAU NDV treated PBMC also induced significant level of early apoptosis on MCF-7 com- 
pared to control (Table 4). Overall, the result indicated that 8 HAU NDV activated PBMC induced utmost cell death on MCF-7 cell by apoptosis. In addition, we analyzed the level of granzyme B with the cytolytic effect of NDV activated PBMC to MCF-7 cell. The level of granzyme B in the media of the co-culture was assayed using ELISA. In the media of MCF-7 cell co-cultured with 8 and 2 HAU NDV treated PBMC, 3.11 and 2.84 fold (which were $287 \mathrm{pg} / \mathrm{ml}$ and 261 $\mathrm{pg} / \mathrm{ml}$ ) increase of granzyme B was detected (Figure $6)$. On the other hand, minimum spontaneous release of granzyme B $(<20 \mathrm{pg} / \mathrm{ml})$ was detected in the NDV treated or untreated PBMC that were not co-cultured with MCF-7. This result indicated that elevated granzyme B expression in NDV treated PBMC may stimulate the apoptosis in MCF-7 cell.

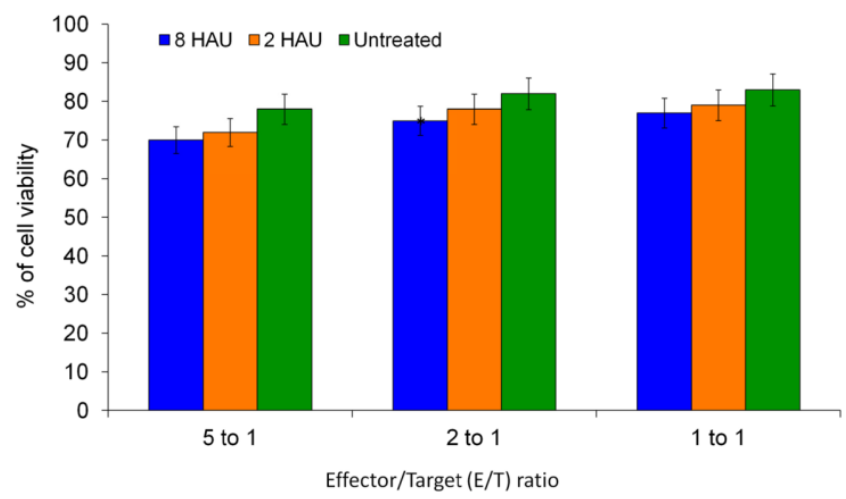

Figure 5. The cell viability of MCF-7 cells (target) after being co-cultured with activated PBMC (effector) at different $E / T$ ratios. The values were the means \pm SE of three independent experiment. The differences between the control group and treated group were determined by one-way ANOVA.

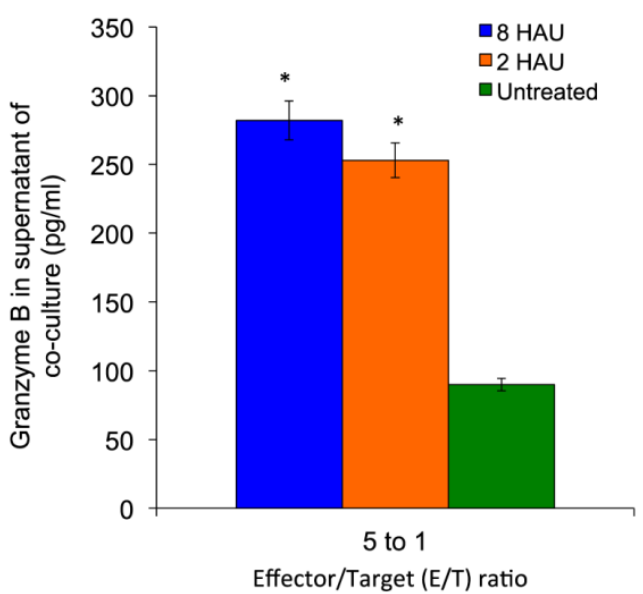

Figure 6. The granzyme B level in supernatant of MCF-7 cells (target) after being co-cultured for 24 hours with activated PBMC (effector) at 5 to I E/T ratios. The values were the means \pm SE of three independent experiment. The differences between the control group and treated group were determined by one-way ANOVA. $(* \mathrm{p}<0.05)$.
Table 4. FACS Annexin-V-FITC/PI apoptosis analysis of MCF-7 cell (Target) after 24 hours co-cultivated with untreated and NDV activated human PBMC (Effector) at 5 to I E/T ratios. The values were the means \pm SE of three independent experiment. The data were analyzed for significance by the statistic software SPSS $(p<$ 0.05).

\begin{tabular}{|c|c|c|c|}
\hline $\begin{array}{l}\text { Virus titer treatment } \\
\text { on PBMC } \\
\text { co-cultured with } \\
\text { MCF-7 }\end{array}$ & $\begin{array}{l}\text { Population of } \\
\text { Viable MCF-7 } \\
(\%) \\
\text { (AnnexinV-/PI-) }\end{array}$ & $\begin{array}{l}\text { Population of } \\
\text { MCF-7 at early } \\
\text { apoptosis }(\%) \\
\left(\text { Annexin } \mathrm{V}^{+} / \mathrm{PI}\right)\end{array}$ & $\begin{array}{l}\text { Population of } \\
\text { MCF-7 at late } \\
\text { apoptosis }(\%) \\
\left(\text { AnnexinV } \mathrm{PI}^{+}\right)\end{array}$ \\
\hline $8 \mathrm{HAU}$ & $57.72 \pm 2.38^{*}$ & $9.49 \pm 2.87^{*}$ & $32.79 \pm 3.33^{*}$ \\
\hline $2 \mathrm{HAU}$ & $63.33 \pm 2.51^{*}$ & $6.42 \pm 3.16$ & $30.25 \pm 2.65^{*}$ \\
\hline Untreated PBMC & $71.34 \pm 3.34$ & $5.10 \pm 2.77$ & $23.56 \pm 1.87$ \\
\hline
\end{tabular}

\section{Discussion}

Immunotherapy which enhances the immune system to fight diseases could be one of the potential approaches for cancer patients. We demonstrated the potential use of NDV local strain AF2240 as an immunomodulator for the human immune system against human breast cancer cells. The virus is expected to work synergistically with chemotherapeutics and immunotherapeutic drugs, as well as surgery and radiotherapy in cancer treatment. In this study, we have shown that the stimulation of all predominant cell populations of PBMCs with low dose of NDV had led to the enhancement of PBMC cytotoxicity. Other experiments directed toward the application of autologous virus modified tumor cell vaccine to cancer patients caused the activation of multiple innate and adaptive immune responses [17]. Several parameters were taken into account including cell proliferation, metabolic activity and cytokine stimulation through activation of immune cells to investigate the immunostimulatory effects of NDV on the human immune system. After the PBMC treatment with NDV, cell proliferation was observed (Figure 1) and treatment with $2 \mathrm{HAU}$ virus titers has expressed the highest proliferative effect. The dynamic of BrdU proliferation was similar with the pattern of IL-2 level indicating that secretion of IL-2 promote the proliferation of PBMC (Figure 1 and 3) since IL-2 was previously reported as being immunoproliferative for cytokine PBMC [18].The human PBMC comprises several effector cells including lymphocytes and NK cells. The virus treated cells exhibiting the cell receptors of CD16 and CD56 increased significantly and reflected the cytolytic NK cell population. In addition, the cytokine secreting NK cells that expressed only CD56 signals were also increased upon virus treatment. Activation of immune cell surface marker is important for the immune cells to enhance the cytolytic activity. CD56 is an isoform of the human neural cell adhesion molecule on human NK cells and it mediates interactions between NK cells and other cells. On the other 
hand, CD16 is one of the activating receptors associated with immunoreceptor tyrosine-based activation motif containing adaptor proteins. CD16 is able to bind to the Fc portions of IgG that further increases antibody-dependent cell-mediated cytotoxicity and helps NK cells to recognize and kill the antibody-coated cells [19]. Crosslinking between target cells and the activating receptors leads to NK cells activation and cytotoxic activity. Activation of lymphocytes and NK cells may induce apoptosis of tumor cells and virus infected cells by granule exocytosis, Fas/Apo-1 and TNF-dependent processes [20]. For the granule exocytosis mechanism, transmembrane channels were generated by direct contact of the PBMC with their target cells resulting in the release of perforin. Together with perforin, granzymes from NK cells enter the MCF-7 target cells and induce apoptosis. In addition, cytokine secretion upon NDV treatment may indicate the activation effect of human PBMC. The activated NK cells upon virus infection can produce IFN- $\gamma$ [20] suggesting the possible involvement of IFN- $\gamma$ in antiviral activity by the NK cells. Based on the ELISA results, high level of IFN- $\gamma$ was obtained upon virus treatment. Releasing of IFN- $\gamma$ promotes TH1 immunity and activates the CD56+ NK cells which augment the antitumor functions [5, 21]. Double staining immunophenotyping showed that the level of perforin and granzymes in the effector cells were increased after being treated with NDV. This suggested that human PBMC was activated by NDV which contributed to the cytotoxic effects on tumor cells. Moreover, cytolytic effect on tumor cells is a parameter to determine the degree of activation on human PBMC. Exposure of PBMC to low dosage of NDV was able to enhance the cytolytic effect against the human breast cancer MCF-7 cell. Based on the MTT result, viability of MCF-7 cells was significantly reduced by activated effector cells. This was further supported by the Annexin V-FITC analysis where the NDV treated PBMC induced both early and late apoptosis on MCF-7 cell. In addition to that, granzyme B was increased 3 fold higher in media of MCF-7 co-cultured with NDV activated PBMC induced apoptosis on MCF-7 cell. Previous reports have demonstrated the success of using NDV for cancer therapy on preclinical mouse in an in vivo study as well as in human clinical trials $[13,22]$. However, NDV may not only have the direct cytotoxic effect on tumor cells but may also be able to enhance the cytolytic activity of immune cells. It has been shown that administration of NDV resulted in stimulation of $\mathrm{T}$ cell responses [23] and NK-mediated cytotoxicity [24]. Besides, the activated NK cell population (CD56+Perforin+/Granzyme B+), none-activated NK cell population (CD56+Perforin-/Granzyme B-) was also found to be stimulated by NDV. It has been reported that the none-activated NK cells secrete large amounts of IFN- $\gamma$ [25]. In the present study, we found increased IFN- $\gamma$ expression and the augmented IFN- $\gamma$ further activated dendritic cells, NK cells and promote $\mathrm{T}$ cell responses [26]. In addition, NDV itself was able to stimulate TNF-a and nitric oxide production to induce antitumor cytotoxicity of macrophages [7].Taken together, NDV strain AF2240 is a potential immunotherapeutic agent that can be used to modulate the human immune system to act against tumor cells. NDV was able to increase proliferation of PBMC, activate the effector surface receptors, stimulate cytokines and cytolytic effect on MCF-7 cells. Further studies should focus on determination of the mechanism of anti-tumor response of isolated subpopulations of human PBMC to substantiate NDV's potential application in tumor immunotherapy.

\section{Acknowledgments}

The authors would also like to thank Professor Tan Soon Guan for proof reading this manuscript. The study was supported by grant 07-05-MGI-GMB 013 from Ministry of Science, Technology and Innovation (MOSTI), Malaysia and a student fellowship from University Putra Malaysia, Malaysia.

\section{Competing Interests}

The authors have declared that no competing interest exists.

\section{References}

1. Kobayashi S, Sato R, Aoki T,et al. Effect of bovine lactoferrin on functions of activated feline peripheral blood mononuclear cells during chronic feline immunodeficiency virus isolation. J Vet Med Sci. 2008; 70: 429-435.

2. De Leeuw O, Peeters B. Complete nucleotide sequence of Newcastle disease virus: evidence for the existence of a new genus within the subfamily Paramyxovirinae. J Gen Virol. 1999; 80: 131-136.

3. Alexander DJ, Jones RC. Paramyxoviridae. In: Poultry Disease 6th ed. Harcourt Publishers Limited: USA. 2008: 294-316.

4. Lorence RM, Rood PA, Kelley KW. Newcastle disease virus as an antineoplastic agent: induction of tumor necrosis factor-alpha and augmentation of its cytotoxicity. J Natl Cancer Inst. 1988; 80: 1305-1312.

5. Sturlan S, Sachet M, Baumann S, Kuznetsova I, Spittler A, Bergmann M. Influenza A virus induces an immediate cytotoxic activity in all major subsets of peripheral blood mononuclear cells. PLoS ONE. 2009; 4: e4122, doi: 10.1371/journal.pone.0004122.

6. [Internet] Velogenic viscerotropic Newcastle disease virus, http://www.cdfa.ca.gov/ahfss/Animal_Health/pdfs/V_V_N_D.pdf

7. Umansky V, Shartrov VA, Lehmann V, Schirrmacher V. Induction of NO synthesis in macrophages by Newcastle disease virus is associated with activation of nuclear factor-Қb. Int Immunol.1996; 8: 491-498.

8. Biron CA, Nguyen KB, Pien GC, Cousens LP, Salazar-Mather TP. Natural killer cells in antiviral defense: function and regulation by innate cytokines. Annu Rev Immunol.1999; 17: 189-220.

9. Yeap SK, Alitheen NB, Ho WY,et al. Immunomodulatory role of Rhaphidophora korthalsii methanol extract on human peripheral blood mononuclear cell proliferation, cytokine secretion and cytolytic activity. J Med Plant Res.2011; 5: 958-965.

10. Chulan U, Ibrahim AL, Babjee AM, Sheikh-Omar AR. Vaccination against Newcastle disease. Trop Anim Health Prod.1982; 14:177-184.

11. Molouki A, Hsu YT, Jahanshiri F, Rosli R, Yusoff K. Newcastle disease virus infection promotes Bax redistribution to mitochondria and cell death in $\mathrm{HeLa}$ cells. Intervirol. 2010; 53: 87-94.

12. Spaeny-Dekking EHA, Hana WL,et al. Extracellular granzyme A and B in humans: detection of native species during CTL responses in vitro and in vivo. J Immunol.1998; 160: 3610-3616. 
13. Krishnamurthy S, Takimoto T, Scroggs RA, Portner A. Differentially regulated interferon response determines the outcome of Newcastle disease virus infection in normal and tumor cell lines. J Virol. 2006; 80: 5145-5155.

14. Yeap SK, Alitheen NB, Ali AM, et al. Effect of Rhaphidophora korthalsii methanol extract on human peripheral blood mononuclear cell proliferation and cytolytic activity toward HepG2. J Ethnopharmacol. 2007; 114: 406-411.

15. CarlensS, Gilljam M, Chambers BJ,et al. A new method for in vitro expansion of cytotoxic human CD3- CD56+ natural killer cells. Hum Immunol. 2001; 62: 1092-1098.

16. Ortaldo JR, Winkler-Pickett R, Wigginton J,et al. Regulation of ITAM-positive receptors: role of IL-12 and IL-18. Blood. 2006; 107: 1468-1475.

17. Schirrmacher V. Clinical trials of antitumor vaccination with an autologous tumor cell vaccine modified by virus infection: improvement of patient survival based on improved antitumor immune memory. Canc Immunol Immunother. 2005; 54: 587-598

18. Brazowski E, Eytan K, Eisenthal A. In vivo modulation of interleukin-2-mediated human peripheral mononuclear cell proliferation and antitumor cytotoxicity by 5-fluorouracil. Anticancer Res. 2007; 27: 4135-4142.

19. Radaev S, Motyka S, Fridman WH, Sautes-Fridman C, Sun PD. The structure of a human type III Fcy receptor in complex with Fc. J Biol Chem. 2001; 276: 16469-16477.

20. Iversen AC, Norris PS, Ware CF, Benedict CA. Human NK cells inhibit cytomegalovirus replication through a noncytolytic mechanism involving lymphotoxin-dependent induction of IFN- $\beta$. J Immunol. 2005; 175: 7568-7574.

21. Reichard KW, Lorence RML, Cascino CJ, et al. Newcastle disease virus selectively kills human tumor cells. J Surg Res.1992; 52: 448-453.

22. Csatary LK, Eckhardt S, Bukosza I, et al. Attenuated veterinary virus vaccine for the treatment of cancer. Cancer Detect Prev.1993; 17: 619-627.

23. Schirrmacher V, Haas C, Bonifer R,et al. Human tumor cell modification by virus infection: an efficient and safe way to produce cancer vaccine with pleiotropic immune stimulatory properties when using Newcastle disease virus. Gene Ther.1999; 6: 63-73.

24. Jarahian M, Watzl C, Fournier P, Arnold A, et al. Activation of natural killer cells by Newcastle disease virus hemagglutinin-neuraminidase. J Virol. 2009, 83: 8108-8121.

25. Ferlazzo G, Munz C. NK cell compartments and their activation by dendritic cells. J Immunol. 2004; 172: 1333-1339.

26. Zeng YJ, Fournier P, Schirrmacher V. Induction of interferon-a and tumor necrosis factor-related apoptosis-inducing ligand in human blood mononuclear cells by hemagglutinin-neuraminidase but not $\mathrm{F}$ protein of Newcastle disease virus. Virology. 2002; 297: 19-30. 